\title{
S48. Schizophrenia: neurobiology, neuropsychology and clinical symptoms
}

\section{SPATIAL MEMORY IN SCHIZOPHRENIA}

\section{LRIZZO. JM DANION. M VAN DER LINDEN*}

Unité INSERM 405 et Département de Psychiatrie Adulte, 1 Place de l'Hôpital, 67091 Strasbourg, France.

* Faculté de Psychologie - Service de Neuropsychologie - B.18 allee du 6 août Sart Tilman 4000 Liège, Belgique.

In order to test the hypothesis that schizophrenia induces an impairment of long-term memory for contextual information, eighteen patients fulfilling DSMIII-R criteria for schizophrenia were compared with eighteen normal control subjects previously matched for sex, age and educational level on a memory task in which words were presented in any one of multiple positions on a grid. One version of the task tapped recognition and recall of the words and a second version tapped two forms of spatial memory, i.e, Iocation-totarget memory and target-to-location memory. Recognition levels of patients and controls were matched by giving patients an increased learning opportunity. Memory for temporal context was also tested, as well as short-term memory, updating working memory, IQ (WAIS), memory quotient (Wechsler Memory Scale) and frontal lobe functions (word fluency test and Wisconsin Card Sorting test). Results will be presented and discussed in relation with the contextual hypothesis of schizophrenia.

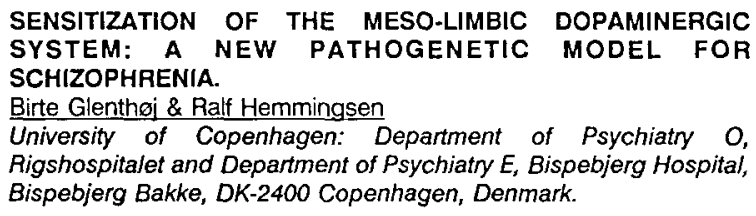
SYSTEM: A NEW PATHOGENETIC MODEL FOR SCHIZOPHRENIA.

Birte Glenthøj \& Ralf Hemmingsen

University of Copenhagen: Department of Psychiatry $O$, Rigshospitalet and Department of Psychiatry E, Bispebjerg Hospital, Bispebjerg Bakke, DK-2400 Copenhagen, Denmark.

We have previously demonstrated that it is possible to modify mesolimbic dopaminergic activity by intermittent electrical stimulations (IES) of rats in the ventral tegmental area (VTA) once daily for 70 days. The intensity of electrical stimulation was determined by the lowest current that elicited a definite motor response. There was a highly significant difference between the current needed to provoke a given response in sensitized rats and in sham stimulated rats. Between the two stimulation periods sensized animals demonstrated a reduced social interaction. To test the effect of early lesions of prefrontal cortex (PFC) on electrical sensitization and on social behaviour, rats with lesions of PFC, stressed rats and control rats were in a later study stimulated in the VTA 4 times daily for 17 days. All rats were electrically sensitized; however, using this shorter lasting sensitization procedure only electrically stimulated rats with prefrontal lesion developed significantly reduced social interaction in social group formation. Neither IES aione, nor lesions of PFC modified social behaviour. The results from our studies demonstrated that sensitization of the meso-limbic cells in the VTA is possible and accompanied by behavioural changes. Based on the new and previous findings a pathogenetic neurobiological hypothesis for the pathogenesis schizophrenia is proposed. We suggest that a primary temporo-limbic defect of glutamatergic receptors lead to an inability to cortical closure of the striato-thalamic "filter" upon activation of the dopaminergic system. The result is a "rigid" striato-thalamic "filter" leaving the subject without possibility to adapt to a changing environment. Repeated demands upon the meso-limbic system will instead result in an altered neuroplastic response, rendering the patient more and more sensitive to environmental stimuli. 
PSYCHOPHARMACOLOGICAL INFLUENCE ON COGNITIVE FUNCTIONING IN SCHIZOPHRENIA

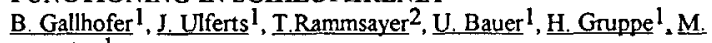
Jantscher ${ }^{1}$

ICentre for Psychiatry, Medical School, Justus Liebig University Giessen, D-35385 Giessen, Germany

${ }^{2}$ Department of Psychology, Justus Liebig University Giessen, Germany

A state of hyperstimulation due to defective sensomotor gating was postulated by Braff and Geyer as a central feature of schizophrenic information processing deficits. To investigate the effect of neuroleptic medication on information processing in schizophrenics we studied 16 healthy subjects, 16 unmedicated schizophrenics and 16 schizophrenics on zotepine, a sedative neuroleptic agent. Sensory and motor reaction times in a series of choice reaction time tasks, thresholds of distinction with regard to a time perception (reference tone $50 \mathrm{~ms}$ ) and a time discrimination task (reference tone $1000 \mathrm{~ms}$ ) as well as the string length of correct answers in a digit span test both forward and backward were measured.

Sensory reaction times were longer in unmedicated schizophrenics than in healthy controls $(\mathrm{p}<.02)$, while medicated schizophrenics did not differ from healthy controls. For the forward version of the digit span no differences between groups were found while both schizophrenic groups showed similarly poor performance compared with healthy controls $(p<.01)$ in the reversed digit span. Healthy controls performed better than unmedicated schizophrenics $(p<.001)$ but did not differ to schizophrenics on zotepine in the $50 \mathrm{~ms}$ range as well as in the $1000 \mathrm{~ms}$ range. Schizophrenics on zotepine showed a better discrimination than unmedicated schizophrenics $(p<.05)$ for both ranges of duration. No major impact on working memory could be derived from schizophrenic disorder, but frontal planning was severely impaired. Zotepine - though being strongly sedative - has led to improvement of some of the tested functions by normalizing cognitve gating mechanisms in the frontal brain. The latter phenomenon supports hyperstimulation theory in schizophrenia.

\section{SUBDIVIDING THE NEGATIVE SYNDROME OF SCHIZOPHRENIA - TOWARDS A MULTI-COMPONENT MODEL OF NEGATIVE SYMPTOMS \\ K Maurer, $H$ Häfner \\ Central Institute of Mental Health, 68159 Mannheim, J5, Germany}

In the literature several dichotomy models of schizophrenia are discussed which subdivide schizophrenia into syndromes of positive and negative symptoms. These models differ in their assumptions concerning the nature of negative symptoms, the relation of positive and negative symptoms as well as the order of these syndromes. Most of these models are based on empirical evidence, but there are also results that do not support them. Therefore it seems useful not simply to consider one model to be the best, refusing the others, but to integrate all of them into a more comprehensive theory. One possibility is to subdivide the negative symptoms syndrome into more specific subsyndromes and to identify several influencing factors that may cause them. An important subdivision of primary disease related deficit symptoms and secondary negative symptoms caused by environmental factors or other psychopathological dimensions of schizophrenia was introduced by Carpenter et al. (1985). However, it seems quite difficult to isolate the components suggested in this model. We will consider several environmental factors (such as coping variables, side effects of neuroleptic drugs, hospitalisation effects) and disease related factors (premorbid personality, duration of acute psychotic episodes, the effect of simultaneous positive symptoms and of depression) and try to estimate the influence of these components on different subdimensions of negative symptoms by developing a path analytical model.

The data to be used for this analysis are from the Mannheim ABCschizophrenia study of 232 first episode schizophrenics, whose early course prior to first hospitalisation was analysed retrospectively using the instrument IRAOS (Häfner et al 1990,1992). A subgroup of 115 of these patients was included in the prospective part of the 5-year follow-up study, whose 3-year follow-up data are available for analysis.

References: Carpenter et al (1985) Schiz Bull 11:440-452; Häfner et al. (1980) Z f Klin Ps 12:230-255; Häfner et al (1992) Schiz Res 6:209-223.
SCHIZOPHRENICS WITH SMALL P300: A SUBGROUP WITH A NEURODEVELOPMENTAL DISTURBANCE AND A HIGH RISK FOR TARDIVE DYSKINESIA?

U Hegerl, G Juckel, A Müller-Schubert, A Pietzcker

Department of psychiatry, Freie Universität Berlin, Eschenallee 3, 14050 Berlin, Germany

It is assumed that structural cortical abnormalities resulting from aberrant brain development in early Iife are an important pathogenetic aspect in a subgroup of schizophrenics. This subgroup is supposed to be characterized by low premorbid adjustment, early onset, incomplete remission, poor outcome, male predominance and high risk for tardive dyskinesia. $A$ reduced P3 (P300) amplitude has to be expected in this subgroup because the laminar and columnar organization of the cortex is of crucial importance for the electrogenesis of P3. Within a 2-year follow-up study on long-term neuroleptic treatment 89 stabilized schizophrenic outpatients were investigated. Both cross-sectional and longitudinal analyses revealed that patients with small P3 have an enhanced risk of developing tardive dyskinesia under neuroleptic treatment. Furthermore, results concerning premorbid adjustment, residual symptoms, relapse rate, and male predominance support the assumption that patients with a reduced $\mathrm{P3}$ correspond clinically to the subgroup which is supposed to have a neurodevelopmental disturbance.

\section{NEUROPSYCHOLOGICAL ASPECTS OF REHABILITATION MEASURES} IN ASYLUM WITH FORCED CURE

L.N. Yurieva

Chair of Psychiatry, Advanced Training Department of Medical Institute, Bechtereva street, 1, 320115 Dniepropetrovsk, Ukraine

The present study is intended to reveal pathobiological pecullarities of schizophrenic patients committed socially hazardous actions and to make use of these findings for individualization of the forced cure, 104 schizophrenic patients (males) with social hazardous actions history were studied with the aid of psychopathological and neuropsychological methods. Neuropsychological investigation was carried out according to the method proposed by A.R. Luria, using quantitative and qualitative analysis of test results. Cerebral dysfunction in different brain regions have been revealed in all patients. Right hemisphere dystunction was the leading squad of the neuropsychological syndrome in $83 \%$ of patients. These changes were strongly pronounced in fronto-temporal region and in the striopallidal system. Obtained data were used along with clinical characteristics for creation of socially oriented rehabilitation programmes. E.g., the best effect was attained in patients with right hemisphere dystunction after the course of treatment by phenothiazine derivatives combined with rational-emotional psychotherapy and occupational therapy connected with image thinking development. The following-up checking has proved the expediency of proposed individual approach to forced cure of schizophrenic patients in special asylums. 
POSITRON EMISSION TOMOGRAPHY STUDY OF CORTICAL 5-HT2 RECEPTORS IN SCHIZOPHRENIA

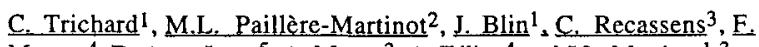
Monnet $^{4}$, D. Attar-Levy ${ }^{5}$, A. Manus ${ }^{3}$, A. Féline ${ }^{4}$ and J.L. Martinot ${ }^{1-3}$

( ${ }^{1}$ INSERM U334, Service Hospitalier F. Joliot, CEA, 4 place du Général Leclerc, 91401 Orsay; ${ }^{2}$ Hôpital de la Salpêtrière, Paris; ${ }^{3}$ Hôpital A. Chenevier, Créteil; ${ }^{4}$ Hôpital de Bicêtre, Kremlin-Bicêtre; ${ }^{5}$ S.H.U. Hôpital Ste-Anne, Paris; France).

Abnormalities of the cortical 5-HT2 receptor densities have been reported in schizophrenia in several post-mortem studies. Particularly, a decrease of 5-HT2 receptor numbers in the frontal cortex of schizophrenic patients has been described, suggesting a dysfunction of the serotoninergic neurotransmission in schizophrenia. However, other post-mortem studies failed to replicate this finding.

We used positron emission tomography and 18-F setoperone, a high affinity radioligand of cortical 5-HT2 receptors, to study the in vivo frontal cortex 5-HT2 binding in a group of untreated schizophrenic patients with respect to a group of sex and age-matched normal controls. Preliminary results of this study, using the frontal to cerebellum ratio method, provide no evidence of a major decrease in the frontal cortex 5 -HT2 receptor density in the patients.

The results obtained in the untreated schizophrenic patients will also be compared to the data observed in a sample of patients treated with amisulpride, a highly selective dopamine receptor antagonist, and with other neuroleptics. 